OPEN ACCESS

Edited by:

Erica Wanless,

University of Newcastle, Australia

Reviewed by:

Ryo Murakami

Konan University, Japan Alexander Francis Routh,

University of Cambridge,

United Kingdom

*Correspondence:

To Ngai

tongai@cuhk.edu.hk

Specialty section:

This article was submitted to

Chemical Engineering,

a section of the journal

Frontiers in Chemistry

Received: 14 February 2018

Accepted: 16 April 2018

Published: 01 May 2018

Citation:

Kwok M and Ngai T (2018) Comparing the Relative Interfacial Affinity of Soft Colloids With Different Crosslinking Densities in Pickering Emulsions. Front. Chem. 6:148. doi: 10.3389/fchem.2018.00148

\section{Comparing the Relative Interfacial Affinity of Soft Colloids With Different Crosslinking Densities in Pickering Emulsions}

\author{
Man-hin Kwok ${ }^{1}$ and To $\mathrm{Ngai}^{1,2 *}$ \\ ${ }^{1}$ Department of Chemistry, The Chinese University of Hong Kong, Shatin, Hong Kong, ${ }^{2}$ Shenzhen Municipal Key Laboratory \\ of Chemical Synthesis of Medicinal Organic Molecules, Shenzhen Research Institute, The Chinese University of Hong Kong, \\ Shenzhen, China
}

Pickering emulsions prepared by various kinds of soft colloids such as the poly(N-isopropylacrylamide) (PNIPAM)-based microgels, have been studied for decades in order to fabricate stimuli-responsive emulsions. It has been generally viewed that the interfacial properties of the microgel monolayers and the emulsion stability are dominated by the softness or deformability of the microgel particles. However, there is still no convenient way to characterize the adsorption/desorption energy of the microgels at the interface although this is an essential topic for microgel-stabilized emulsions. This paper presents a novel method for directly comparing the relative interfacial affinity of microgel particles with comparable size but different crosslinking densities, therefore, different softness at the oil/water interface. Typical micron-sized PNIPAM-based microgels were synthesized and used in this study. With advanced fluorescent labeling techniques, we are capable of distinguishing different kinds of microgels in a Pickering emulsion. During vigorous agitation, particles with higher adsorption energy are more likely to be found at the oil/water interface instead of the loosely adsorbed counterparts. By counting the ratio of interfacial area occupied by two microgels, the interfacial affinity of them can be compared. It is found that interfacial affinity of microgels is not only dependent on the softness but also strongly correlated with the core-shell morphology of the microgels, especially the outmost collapsed polymer layer at the interface. This result is consistent with the interfacial morphology model proposed by other researchers. The understanding of the stabilization of such Pickering emulsions can help us to design and develop responsive Pickering emulsions with better controlled stability.

Keywords: Pickering emulsions, microgels, PNIPAM, soft colloids, microgel-stabilized emulsions

\section{INTRODUCTION}

Pickering emulsion was first described by Ramsden (1904) and S. U. Pickering more than 100 years ago (Pickering, 1907). Submicron or micron-sized solid particles like surfactants or amphiphilic polymers, can adsorb at oil-water interfaces, and provided long-term kinetic stability. Such particlestabilized emulsions are now commonly called Pickering (or Pickering-Ramsden) emulsions. These stabilizing particles are wetted by both phases in the system and they are interfacially active. 
Unlike the conventional small molecule surfactants, the particle stabilizers are usually considered to be irreversibly adsorbed to the interface and cannot be removed by thermal energy (Schmitt and Ravaine, 2013).

Since the early 1900's studies on solid particles at interfaces, the issue of correlating the properties of individual particles at the interface with emulsion stability has remained largely unexplored until the last couple of decades. Nevertheless, with the advancement in preparation of various kinds of colloidal particles, the topic has attracted so much more attention in physical science research (Binks, 1998; Chen et al., 2007; Li and Stover, 2008; Liu et al., 2008; Tsuji and Kawaguchi, 2008; Richtering, 2012; Destribats et al., 2014; Style et al., 2015). Pickering emulsions retain the basic properties of classical emulsions stabilized by surfactants or proteins so that they can be substituted for classical emulsions in most industrial and technological applications. Moreover, Pickering emulsions offer several remarkable advantages over conventional surfactantstabilized emulsions, such as high resistance to coalescence and reduced foaming (by hydrophobic particles) (Aveyard et al., 1994). The "surfactant-free" character makes them more attractive in personal care and pharmaceutical applications where surfactants often cause adverse effects such as irritancy and even cell damage (Tang et al., 2015). Therefore, they have received intense attention in the past decade.

Besides hard spherical particles, Pickering emulsions stabilized by soft polymeric particles have also been developed (Ngai et al., 2005, 2006). Particles made of soft matter are able to significantly change their properties when they are triggered by external stimulations, such as temperature (Pelton and Chibante, 1986) pH (Hoare and Pelton, 2004, 2008; Khan, 2007) ionic strength (Saunders and Vincent, 1999) or even magnetic field (Khan, 2008). Therefore, the use of soft particles in stabilizing Pickering emulsions allows a convenient way to prepare responsive emulsions, which are also known as "smart emulsions." The responsiveness of the soft particles can be transferred to the corresponding Pickering emulsions. The development of such responsive emulsions leads to even more potential applications, for example, biocatalysis (Wiese et al., 2013), oil transportation ( $\mathrm{Li}$ and Stover, 2008), oil refinery (Brugger et al., 2008), and drug delivery (Frelichowska et al., 2009; Zhang et al., 2010; Chevalier and Bolzinger, 2013).

Whilst soft particles have been demonstrated as being interesting stabilizers for Pickering emulsions, the mechanism and detail of the stabilization given by these soft particles are still not fully understood. In the past few years, many reports studied Pickering emulsions stabilized by soft particles, especially poly( $N$-isopropylacrylamide) (PNIPAM)-based microgel particles (Brugger et al., 2010; Geisel et al., 2012, 2014a,b; Destribats et al., 2013, 2014; Monteillet et al., 2014; Pinaud et al., 2014). The softness or the deformability of microgels has been emphasized to play an important role in the stabilization of emulsions. For example, Destribats et al. (2011) obtained the images of PNIPAM-based microgel particles at the oil/water interface using cryo-scanning electron microscopy (cyro-SEM) techniques. Based on their SEM images, they concluded that microgel particles are often deformed and stretched at the interface. They described the conformation as "fried egg-like structure" and suggested that the deformability of the microgel particles was important in stabilizing the corresponding Pickering emulsions. It is reasonable to attribute the high stability of the emulsion to the deformability of the stabilizers because the flattening of microgel particles would increase the coverage of each particle and form a better and elastic protecting layer.

However, in our recent study, we observed individual, micronsized microgel particle at the oil/water interface under confocal laser scanning microscopy (CLSM) (Kwok and Ngai, 2016). It was found that the deformation of the overall shape of micronsized microgel is not significant. Compared with cryo-SEM, CLSM does not offer images with very high resolution but the images can be taken in aqueous solution, the native state instead of high vacuum, dried state of soft particles. Therefore, CLSM is likely a better choice for characterizing these water swollen gel particles. We argued that larger microgel only significantly deform at extremely swollen condition, which refers to the $\mathrm{pH}$-responsive swelling. For PNIPAM microgel without $\mathrm{pH}$-responsiveness, the corresponding deformation might not be significant as shown in cryo-SEM of the sub-micron-sized microgels.

Besides our CLSM images, Geisel et al. obtained images of microgel-stabilized Pickering emulsions in aqueous state using novel transmission X-ray microscopy (Geisel et al., 2014a). In their images, the main body of the particles do not show any significant flattening or deformation. Nevertheless, deformation near the interface is found. Recently, Style et al. took cryo-SEM images of a fractured water-decane interface populated by PNIPAM microgel particles with good resolution (Style et al., 2015). In this peculiar side-view as shown in Figure 1, soft microgel particles show asymmetric conformations across the interface, with two different sizes and shapes of the particle portions exposed to the two fluids. However, it can be clearly found that the main part of the microgel particle is not significantly deformed. It seems that the oil/water interface in between the particles is covered by a layer of the collapsed polymer which is connected into networks. Their results are consistent with our previous confocal results and the measurement of the elastic modulus of PNIPAM-based microgel particles from other AFM based studies (Hashmi and Dufresne, 2009; Burmistrova et al., 2011; Kwok and Ngai, 2016). Moreover, Zielinska et al. have recently used neutron reflectivity to study the PNIPAM-based nanogels at the water/air interface (Zielinska et al., 2016). They found that the nanogels at the interface have a collapsed polymer layer in contact with air. This collapsed polymer layer has a low water content which is similar to that for a collapsed microgel at temperatures above the volume transition temperature (VTP). However, it is still an open question how the morphology of the microgels and this outermost collapsed polymer layer influence the adsorption/desorption energy of individual microgel particles at the interface which is not easy to be measured. In order to connect interfacial properties between soft particles and emulsion stability, in this study, we have developed a novel method to compare the relative interfacial affinity or surface activity of the microgels with different 


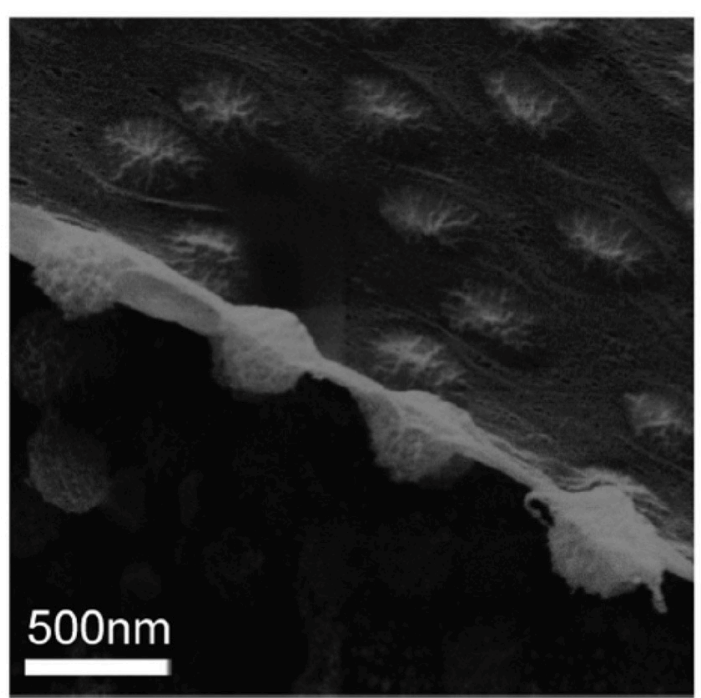

FIGURE 1 | Cryo-SEM image of a fracture water-decane interface populated by PNIPAM microgel particles (Style et al., 2015). Reproduced with permission of The Royal Society of Chemistry.

softness. PNIPAM-based microgels with different crosslinking densities were firstly synthesized and mixed together to stabilize emulsions. By using excess microgel particles, the number of microgels at the oil/water interface was no longer limited by the total number of particles. Instead, the number of a specific microgel sample populated at the interface depended on its affinity to the interface, which directly reflected its desorption energy. With optimized labeling techniques, microgels with different softness within the same emulsion sample can be distinguished clearly. Combining with the deformation model suggested by other literatures, which also matches our previous CLSM images, the stabilization of soft microgels with different softness and morphology on resulting Pickering emulsion can be explained. By keeping the sizes of our microgel samples the same, we found that the interfacial affinities of the microgel stabilizers are not only dependent on the crosslink density but also strongly correlated with the outermost collapsed polymer layer of the microgel in controlling the emulsion stability. The results presented in this paper bring new insights for controlling the stability of Pickering emulsions, particularly using soft colloids as stabilizers.

\section{MATERIALS AND METHODS}

\section{Materials}

$\mathrm{N}$-isopropylacrylamide (NIPAM, Fluka) was recrystallized using a 1:1 toluene/n-hexane mixture twice. $N, N^{\prime}$-Methylenebisacrylamide (MBA, Fluka) was recrystallized using methanol. Potassium persulfate (KPS, Merck), decane (Sigma Aldrich), methacryloxyethyl thiocarbonyl rhodamine B (RB, Polysciences), and fluorescein sodium salt (FSS, Sigma Aldrich) were used as received. Milli-Q deionized water was used in all the experiments.

\section{Preparation of PNIPAM Microgels}

The procedures to prepare micrometer-sized microgel particles with tailored structure and different cross-linker contents have been documented in our previous publication (Kwok et al., 2013). We briefly describe different types of microgels prepared for this work as below.

\section{PNIPAM Microgels of Diameter Around $1.4 \mu \mathrm{m}$ With $10 \mathrm{mg}$ MBA Cross-Linker}

First, $1.0 \mathrm{~g}$ of NIPAM, $1 \mathrm{mg}$ of RB, and $0.01 \mathrm{~g}$ of MBA were dissolved in $90 \mathrm{ml}$ deionized water and filtered to remove any solid impurities. The solution was then transferred to a $250 \mathrm{ml}$ round-bottomed flask. The solution was purged with nitrogen gas and the solution was stirred in a $43^{\circ} \mathrm{C}$ water bath for $1 \mathrm{~h}$ so that the dissolved oxygen was removed. Then, $0.09 \mathrm{~g}$ KPS was dissolved in around $3 \mathrm{ml}$ deionized water and added to the reaction vessel with a syringe for initiation of the polymerization. Once the solution started to turn opalescent, which typically happened within 4-6 min, the temperature was immediately ramped to $60^{\circ} \mathrm{C}$ with a constant ramp rate of $2^{\circ} \mathrm{C} / \mathrm{min}$. Finally, the reaction mixture was stirred for $3 \mathrm{~h}$ at $60^{\circ} \mathrm{C}$. This sample was labeled as L10 (meaning large size of microgel with $10 \mathrm{mg}$ of MBA). Three more microgels with diameters also around $1.4 \mu \mathrm{m}$ were synthesized with similar procedures but without the RB fluorescent labeling. Their specific conditions and sample names were shown in Table 1. L50A was prepared by the same procedures as L50, except that after the temperature reached to $60^{\circ} \mathrm{C}$ for $30 \mathrm{~min}$, an extra $10 \mathrm{mg}$ of $\mathrm{MBA}$ was added to the reaction mixture. The addition of $\mathrm{MBA}$ at the late stage is for cross-linking the dangling chains on the periphery of the microgel particles.

\section{PNIPAM Microgels of Diameter Around $900 \mathrm{~nm}$ With $30 \mathrm{mg}$ MBA Cross-Linker}

Similar to the procedures of synthesizing L30, the monomer solution was prepared, but with a volume of $60 \mathrm{ml}$ and $1 \mathrm{mg}$ of $\mathrm{RB}$ dissolved in the solution. After $0.05 \mathrm{~g}$ of KPS was added to the reaction vessel at $55^{\circ} \mathrm{C}$, the temperature was immediately ramped to $70^{\circ} \mathrm{C}$ in half an hour. Finally, the reaction mixture was stirred for $3 \mathrm{~h}$ at $70^{\circ} \mathrm{C}$. This sample was called M30.

All of the synthesized microgels were purified by centrifugation in order to remove any unreacted monomers, oligomer chains and the unreacted initiator. The microgels were

TABLE 1 | The experimental conditions for synthesizing L10, L30, L50, L80 PNIPAM micogel particles.

\begin{tabular}{lllc}
\hline Sample name & MBA content & $\begin{array}{l}\text { Reaction } \\
\text { temperature }\end{array}$ & Volume of solution \\
\hline L10 & $10 \mathrm{mg}$ & $43^{\circ} \mathrm{C}$ & 90 \\
L30 & $30 \mathrm{mg}$ & $43^{\circ} \mathrm{C}$ & 110 \\
L50 & $50 \mathrm{mg}$ & $42^{\circ} \mathrm{C}$ & 120 \\
L50A & $50 \pm 10 \mathrm{mg}$ & $42^{\circ} \mathrm{C}$ & 120 \\
L80 & $80 \mathrm{mg}$ & $40^{\circ} \mathrm{C}$ & 140
\end{tabular}

Underline values indicates " $50+10 \mathrm{mg}$ " -50 mg of MBA was used initially and $10 \mathrm{mg}$ of MBA was added in the middle of the reaction. 
purified at a constant maximum centrifugal force of $28,000 \mathrm{~g}$ for $1 \mathrm{~h}$. After that, the supernatant was removed and the microgels were dispersed again in deionized water (or microgel solution for concentrating the sample) by stirring overnight. The purification cycle was repeated four times for each of the samples.

\section{Physical Measurements Laser Diffraction Measurement}

Deionized water was used to fill up the sample chamber of the Coulter LS230 laser diffraction size analyser. Background measurements and detector alignment were done by the provided software. Then $1 \% \mathrm{wt} / \mathrm{wt}$ microgel samples were added to the analyzer and the measurements of the particle sizes were performed.

\section{Concentration Determination}

The mass of a clean glass vial was recorded accurately by an analytical balance. After that, about $0.5 \mathrm{~mL}$ of the purified microgel sample was transferred to the glass vial, and the total mass of it was measured carefully. Then the glass vial was put in an oven at $150^{\circ} \mathrm{C}$ to evaporate the water. After the vial was cooled to room temperature, the total mass of the residue and the vial was measured again. Finally, the concentration of the microgel was calculated as a weight percentage.

\section{Pickering Emulsion Stability Measurement}

$0.7 \mathrm{~mL}$ of decane was added to $0.7 \mathrm{~mL}$ of $1.0 \% \mathrm{wt} / \mathrm{wt}$ microgel solution. Then, the emulsion was prepared by an Ultra Turrax T25 homogenizer (with $10 \mathrm{~mm}$ head) operating at 9,500 rpm. After that, the emulsion was placed in a centrifuge for $30 \mathrm{~min}$. The centrifugal force was set at 1,000 g. The centrifugation was repeated until the oil released did not change anymore. Finally, a photo of the emulsion after centrifugation was taken to measure the oil released.

\section{Relative Interfacial Affinity of Different Microgels}

Fifty microliters of decane was added to $1 \mathrm{~mL}$ of the $1 \% \mathrm{wt} / \mathrm{wt}$ mixed microgel solution $0.30 \mu \mathrm{L}$ of $0.3 \mathrm{mg} / \mathrm{mL}$ FSS solution, and $10 \mu \mathrm{L}$ of $0.075 \mathrm{M}$ sulfuric acid were also added. Then, the emulsion was prepared by the homogenizer operating at 9,500 rpm for $2 \mathrm{~min}$. CLSM images of the emulsion were taken with a Nikon Eclipse Ti inverted microscope (Nikon). The wavelength of the excitation laser for FSS and RB were $488 \mathrm{~nm}$ and $543 \mathrm{~nm}$ respectively. A $60 \times(\mathrm{NA}=1.49)$ oil immersion objective was used. Images were taken from many different portions of the emulsion.

\section{RESULTS AND DISCUSSION}

\section{Microgel Preparations and Characterizations}

To synthesize the required microgel particles, surfactant free emulsion polymerization (SFEP), which is also known as precipitation polymerization, was applied. Figure 2 shows schematics of the syntheses. It is found that among numerous synthetic parameters, cross-linker content, nucleation temperature and the total monomer concentration are the key parameters for controlling the particle size. All of the syntheses in this work were based on batch synthesis. The monomers were all added to the reaction mixtures, except for sample L50A. It is worth noting that many reports have indicated that batch polymerization at high temperature can result in a poorly controlled microgel network structure since the cross-linker MBA monomer was commonly incorporated into the microgels faster than the NIPAM monomer. This suggests that the microgel particles prepared in this study would have a core-shell morphology with a highly cross-linked core surrounded a shell of dangling polymer chains. We used only one such kind of particle morphology because the Pickering emulsions stabilized by microgel particles are complicated. Therefore, the comparison of emulsion stabilities was limited to only one variable, the total cross-linker content or softness of the microgels.

For the syntheses of micron-sized microgels with different cross-linking densities (samples named as L10, L30, L50, L80, L50A), temperature-programmed emulsion polymerization was applied. Large PNIPAM particles can be prepared at lower temperature, which is typically around $45^{\circ} \mathrm{C}$. However, the yield of the reaction is relatively low and a lot of oligomeric chains, not involved in particle growth, will be formed. Therefore, applying a temperature ramp right after the nucleation state can produce stable micron-sized microgel dispersions with reasonable yield. High cross-linking density on the other hand prevents the microgel from dissolving in water at low temperature. Therefore, it was important to note that the temperature of each synthesis was slightly different. In order to prepare microgels with different cross-linker contents and similar diameter, L10 was prepared at a slightly higher temperature so that the size of it could be reduced and L80 was prepared at a slightly lower temperature. The volume of reaction mixtures was different so that aggregation in the syntheses could be minimized.

We used a laser diffraction particle size analyser and dynamic light scattering (DLS) to characterize the diameters of the synthesized PNIPAM microgels. Figure 3 shows the laser diffraction measurements of the microgels. The size distributions of large microgels were very similar, with means around $1.4 \mu \mathrm{m}$. In Table 2, the size measurements of all five microgels were summarized. We calculated the thermal responsive swelling ratios $(\mathrm{Q})$ by dividing the diameters measured by DLS $\mathrm{D}_{\mathrm{h}}$ at $25^{\circ} \mathrm{C}$ with $\mathrm{D}_{\mathrm{h}}$ at $40^{\circ} \mathrm{C}$. This swelling ratio was affected by the softness of the particles. The swelling ratio increased as the softness of the particle increased. From the diameter swelling ratios shown in Table 2, it was found that the difference in diameter swelling ratios between L10 and L80 were consistent with the corresponding cross-linker content.

\section{Determining the Stability of the Pickering Emulsions Stabilized by Microgels Using Centrifugation}

For measuring the stability of the microgel stabilized emulsion, centrifugation was applied as it is a widely utilized method to quantitatively measure emulsion stability. The advantage of this method relies in the fact that it is direct and easy to perform. The method determines the maximum pressure which can be 

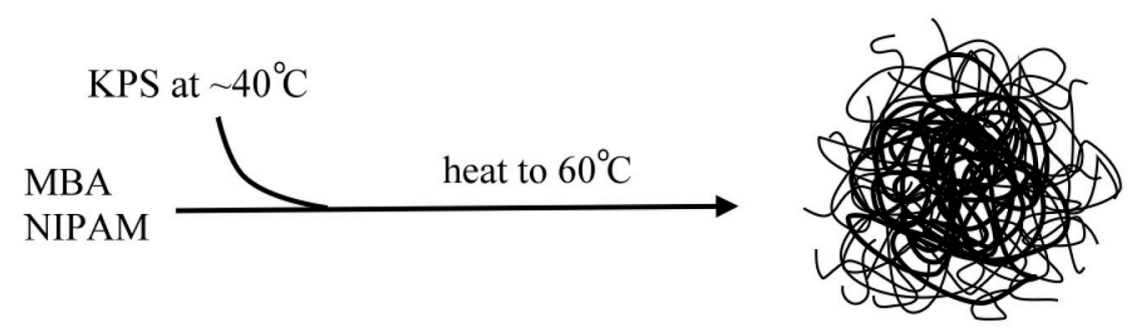

Micron-sized batch microgel

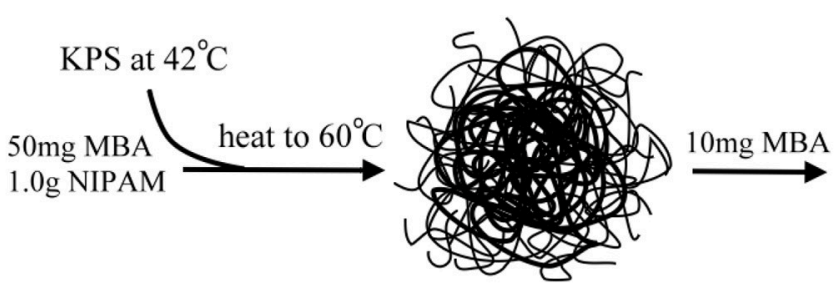

reacts for $0.5 \mathrm{hr}$

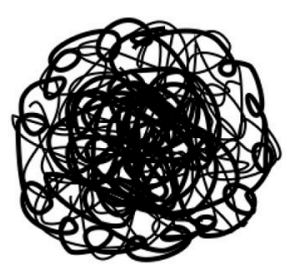

L50A

FIGURE 2 | Schematic illustration of the preparation of the micron-sized PNIPAM-based microgel samples.

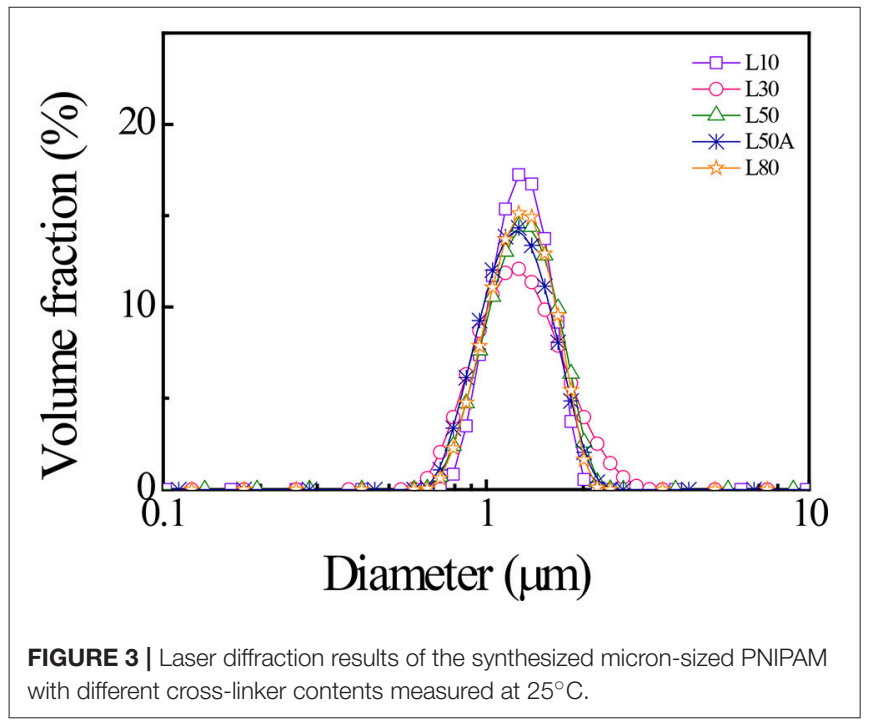

withstood by the water thin film between oil droplets before coalescence occurs. This pressure is called the maximum osmotic pressure $P_{\text {osm }}$. Campbell et al. have suggested that this maximum osmotic pressure is a complete analogy with the maximum capillary pressure (Tcholakova et al., 2002). Therefore, emulsions with a higher $P_{\text {osm }}$, are able to resist coalescence for a longer period of time, the emulsions are thus more stable. To calculate this $P_{\text {osm }}$ from the centrifugation data, the following equation was used:

$$
P_{\text {osm }}=\Delta \rho g_{\max }\left(H_{o i l}-H_{r}\right)
$$

TABLE 2 | The $D_{h}, D_{L D}$ and the corresponding VPT diameter swelling ratios (Q) of the PNIPAM microgels.

\begin{tabular}{lrrrr}
\hline Sample & $\mathbf{D}_{\mathbf{h}}(\mathbf{n m})$ at $\mathbf{2 5}^{\circ} \mathbf{C}$ & $\mathbf{D}_{\mathbf{L D}}(\mathbf{n m})$ at $\mathbf{2 5} \mathbf{C}^{\circ} \mathbf{C}$ & $\mathbf{D}_{\mathbf{h}}(\mathbf{n m})$ at $\mathbf{4 0} \mathbf{}^{\circ} \mathbf{C}$ & $\mathbf{Q}$ \\
\hline M30 & $960 \pm 110$ & $940 \pm 270$ & $408 \pm 60$ & 2.3 \\
L10 & $1400 \pm 240$ & $1360 \pm 260$ & $470 \pm 46$ & 3.0 \\
L30 & $1450 \pm 240$ & $1390 \pm 420$ & $518 \pm 80$ & 2.8 \\
L50 & $1400 \pm 260$ & $1380 \pm 320$ & $518 \pm 86$ & 2.7 \\
L50A & $1350 \pm 210$ & $1330 \pm 310$ & $504 \pm 79$ & 2.7 \\
L80 & $1370 \pm 220$ & $1360 \pm 300$ & $522 \pm 101$ & 2.6 \\
\hline
\end{tabular}

In this equation, $g_{\max }$ is the maximum centrifugal acceleration; $\Delta \rho$ is the density difference between the oil and water; $H_{r}$ is the height of the oil released by the centrifugation process, and $H_{o i l}$ is the height of the oil when there is total phase separation.

Figure 4 shows the emulsion stability measurements of the large microgel stabilized emulsions after centrifugation at $1,000 \mathrm{~g}$. Although the energy of the particle desorption is usually a few orders larger than the centrifugal potential energy, it was essential to further confirm that the centrifugal force was not large enough to actively remove microgel particles from the oilwater interface. Therefore, the centrifugation was repeated and it was found that the amount of oil released was unchanged after a few centrifugations.

In Figure 4, it can be seen that the stability of the emulsion decreased as the MBA content increased for the microgel stabilized emulsion samples. Note that sample L50A was an exception. By comparing the maximum osmotic pressures of emulsions were stabilized by L50 and L80 with L50A, we found 


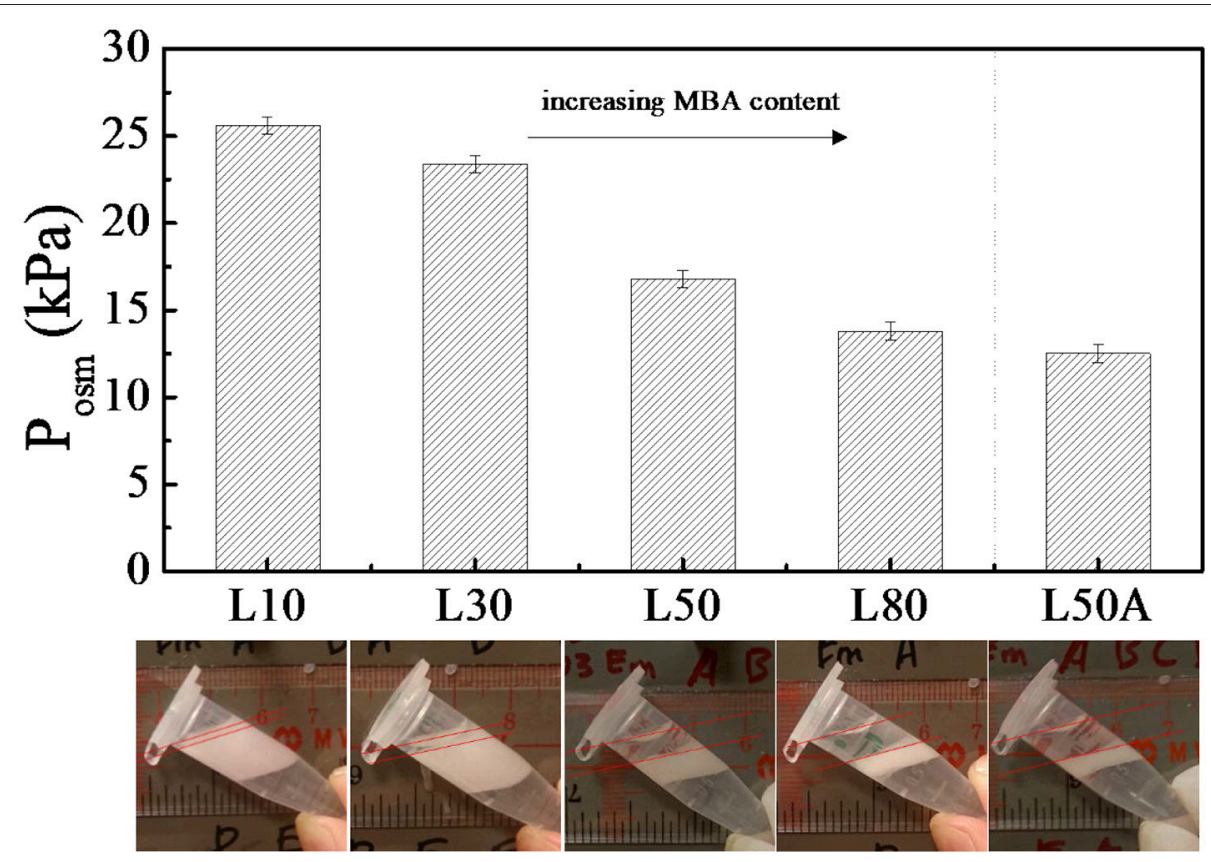

FIGURE 4 | The maximum osmotic pressure ( $\left.P_{\text {osm }}\right)$ of emulsions stabilized by large microgels with different cross-linker contents. The centrifugation was done at $1,000 \mathrm{~g}$ ( $\mathrm{g}$ is gravitational acceleration). The images below the bars are the corresponding photos of the emulsion samples after centrifugation.

that L50A stabilized emulsions were the least stable. However, L50 and L50A just differed by the surface property and L80 possessed even more MBA content that L50A. We will discuss more about this stability difference in the next section.

\section{Comparison of Surface Affinity Between Microgels With Different Size}

It is often suggested that Pickering emulsion stability is strongly related to the interfacial energy of the particles because they reduce the area of the interface between the two immiscible liquid. Also, the energy is usually a few orders of magnitude larger than thermal energy, which leads to an ultra-strong anchoring of particles at the interface. The energy required when a single rigid spherical particle was desorbed from the interface is given by:

$$
\Delta E=\gamma \pi r^{2}(1-|\cos \theta|)^{2}(\text { ChevalierandBolzinger, 2013) }
$$

where $r$ is the radius of the particle, $\gamma$ is the surface tension and $\theta$ is the contact angle of the particle. This desorption energy is affected by many factors, such as size, contact angle, roughness, etc. If the particles are adsorbed to the interface with higher energy, they form a particle shell around the emulsion droplet with higher strength and the shell is capable of stabilizing the emulsion better. Therefore, the desorption energy is one of the crucial factors in Pickering emulsion stability and this energy can be compared by the surface affinity of the particle. The contact angle of microgel at the oil-water interface was around $40^{\circ}$, which was estimated by the effective contact angle of Richtering's work
(Geisel et al., 2012). Therefore, the desorption energy of our larger microgels is around $1.9 \times 10^{6} \mathrm{k}_{\mathrm{B}} \mathrm{T}$.

CLSM is a chosen for this study because the sample preparation is much easier and there is almost no disturbance to the emulsion sample. Also, the emulsion can be visualized in solution instead of vacuum as commonly viewed by electron microscopy. To compare the relative surface affinity of different microgel particles, we mixed two microgel samples, which were labeled differently, and prepared the emulsion with the homogenizer after adding oil. CLSM images were taken and the number of each particle type at the oil-water interface counted. We call this number ratio $\phi$. As the diameters of the particles were well-characterized, the relative surface coverage, which is defined to be our relative interfacial affinity $\Phi$ can be easily calculated. The method is based on the equilibrium established by the two kinds of microgel and the energy input by the homogenizer. With rigorous agitation, individual microgel particles in the bulk solution are capable of displacing another particle which has been adsorbed at the interface. The probability of this process is depended on their relative desorption energy. A particle with lower desorption energy is less likely to displace a particle with higher desorption energy and vice versa. Therefore, higher interfacial affinity indicated higher desorption energy of the particles.

We synthesized microgel M30, which has diameter of around $900 \mathrm{~nm}$ and polymerizable red fluorescent dye, RB, was also added in the synthesis. The reason that we used sample M30 instead of L10 in the demonstration was because RB could be much better incorporated into the microgel at high temperature synthesis so that the image quality was better. In Figure 5, we can clearly see that M30 and L30 showed different colors under 


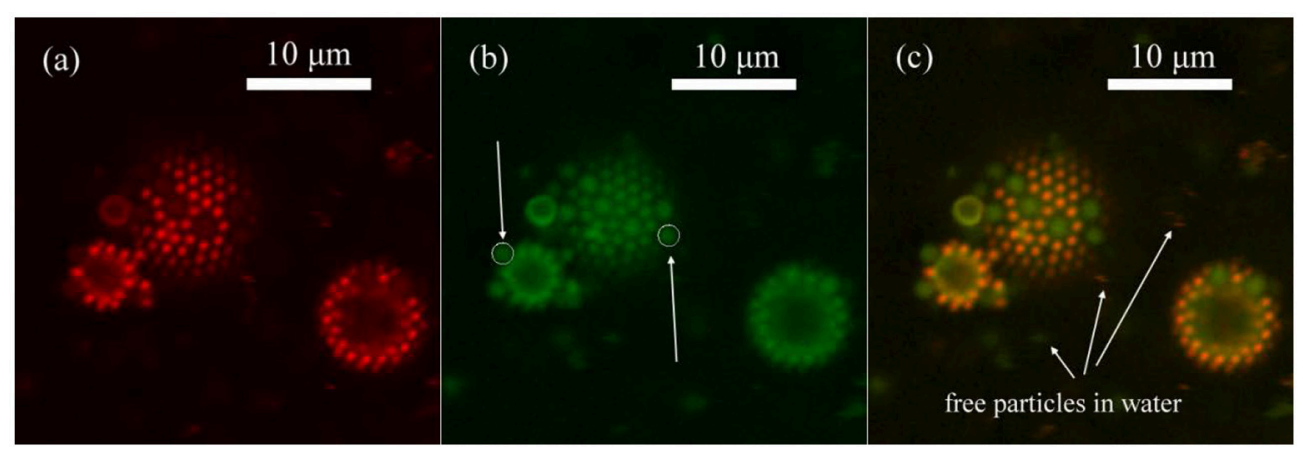

FIGURE 5 | CLSM image of emulsion prepared by M30 and L30 microgels in 1:1 weight concentration ratio in bulk (a) Red channel of Rhodamine B fluorescence, which was only given by M30 microgels. (b) Green channel of fluorescein fluorescence, which was given by M30 and also L30 microgels. (c) Combined image of both channels. Free M30 and L30 microgel particles presented in water, indicating that microgel particles were in excess in the system.

CLSM. M30 is orange and L30 is mostly green. In the synthesis of $\mathrm{M} 30, \mathrm{RB}$ was added so that the red fluorescent dye was covalently bonded onto the M30 microgel. Next, both of the microgels were labeled by diffusing fluorescein (from fluorescein sodium salt). The adsorption of fluorescein to the microgel was based on the H-bond interaction. (Kwok et al., 2013) Therefore, the two microgels were clearly distinguishable at the oil-water interface. The initial bulk ratio (weight concentration) between M30 and L30 was 1:1. To obtain a statistically valid result, over 4,000 particles were counted from different portions of the emulsion.

In addition, for the smaller emulsion droplet in Figure $\mathbf{5 b}$, the CLSM image was taken near half of the height of the droplet. On the other hand, the CLSM image of the middle sized droplet in Figure $\mathbf{5 b}$ was taken near the bottom of the droplet. A white circle of the same size was put onto one L30 particle on each droplet. Obviously, the two L30 particles were identical in either shape or size under CLSM. Therefore, we also could confirm that the main body of the particles were not deformed significantly.

In Figure 5c, we can see that there were free M30 and L30 microgel particles, which were not adsorbed at the interface, in the bulk solution. This is because only a very small amount of oil was used to prepare the emulsion. Both M30 and L30 were in excess while the emulsion was prepared under the vigorous stirring of the homogenizer. In order to further confirm that the current CLSM image results were representing the equilibrium distribution between M30 and L30 on the interface, we prepared the emulsion in four different ways. The initial bulk ratio between M30 and L30 was changed to 2:1 and 1:2, respectively. Also, the emulsion was first prepared by M30 (or L30), then L30 (or M30) was added and the homogenizing process repeated. Table 3 summarizes all five results and the results of (A), (B), (D), and (E) were statistically the same. Result (D) and (E) showed that under homogenization, particle desorption and displacement was allowed. Both M30 and L30 microgel particles were capable of displacing each other from the oil-water interface. Therefore, these particle counting results were not kinetically controlled by the initial adsorption. From result (A) and (B) shown in Table 3, it was confirmed that both M30 and L30 microgels were in excess at an initial ratio of $1: 1$ and 2:1. It was because the excess particles
TABLE 3 | Particle counting results of M30 and L30 at the interface.

\begin{tabular}{|c|c|c|c|c|}
\hline Image no. & 1 & 2 & 3 & Total \\
\hline \multicolumn{5}{|c|}{$\begin{array}{l}\text { (A) M30 TO L30 WT. CONC. RATIO = 1:1 (\# RATIO = 3.4: 1), } \\
\text { EMULSION PREPARED BY ONE STEP }\end{array}$} \\
\hline No. of M30 & 701 & 3,776 & 1,863 & 6,340 \\
\hline No. of L30 & 127 & 672 & 329 & 1,128 \\
\hline M30: L30 at interface & 5.52 & 5.62 & 5.66 & $5.62 \pm 0.05$ \\
\hline \multicolumn{5}{|c|}{$\begin{array}{l}\text { (B) M30 TO L30 Wt. CONC. RATIO = 2:1 (\# Ratio = 6.8:1), } \\
\text { EMULSION PREPARED BY ONE STEP }\end{array}$} \\
\hline No. of M30 & 1,633 & 1,702 & 1,380 & 4,715 \\
\hline No. of L30 & 290 & 302 & 249 & 841 \\
\hline M30: L30 at interface & 5.63 & 5.64 & 5.54 & $5.61 \pm 0.05$ \\
\hline
\end{tabular}

\section{(C) M30 TO L30 WT. CONC. RATIO = 1:2 (\# RATIO = 1.7: 1),} EMULSION PREPARED BY ONE STEP

$\begin{array}{lllll}\text { No. of M30 } & 625 & 1,459 & 1,179 & 3,263\end{array}$

$\begin{array}{llll}\text { No. of L30 } & 215 & 513 & 421\end{array}$

$\begin{array}{llll}\text { M30: L30 at interface } \quad 2.90 & 2.84 & 2.80 & 2.84 \pm 0.05\end{array}$

\begin{tabular}{|c|c|c|c|c|}
\hline \multicolumn{5}{|c|}{$\begin{array}{l}\text { (D) M30 TO L30 WT. CONC. RATIO = 1:1 (\# RATIO = 3.4: 1), } \\
\text { EMULSION PREPARED BY M30 FIRST }\end{array}$} \\
\hline No. of M30 & 1,912 & 1,759 & 1,089 & 4,760 \\
\hline No. of L30 & 351 & 305 & 196 & 852 \\
\hline M30: L30 at interface & 5.45 & 5.77 & 5.56 & $5.59 \pm 0.17$ \\
\hline
\end{tabular}

\begin{tabular}{|c|c|c|c|c|}
\hline \multicolumn{5}{|c|}{$\begin{array}{l}\text { (E) M30 TO L30 WT. CONC. RATIO = 1:1 (\# RATIO = 3.4: 1), } \\
\text { EMULSION PREPARED BY L30 FIRST }\end{array}$} \\
\hline No. of M30 & 1,066 & 1,455 & 2,415 & 4,936 \\
\hline No. of L30 & 191 & 261 & 431 & 883 \\
\hline M30: L30 at interface & 5.58 & 5.57 & 5.60 & $5.59 \pm 0$ \\
\hline
\end{tabular}

The overall ratio is the average of $\varphi$ and its weighted standard deviation. The initial bulk \# ratio was also calculated based on the assumption that $M 30$ and L30 have the same density.

in the solution did not affect the equilibrium of the adsorption of different particles at the interface. However, from result (C) in Table 3, it was believed that at a M30 to L30 ratio of 1:2, M30 microgel was limited in the system, so that the excess interface was covered by L30 instead. Hence, result (C) in Table 3 was smaller than results (A) and (B).

We determined the equilibrium number ratio $\phi$ of $M 30$ to L30 on the oil-water interface to be 5.62 at $9,500 \mathrm{rpm}$ 
stirring, from result (A) in Table 3. However, it was important to note that small and large particles occupied different areas at the interface. Therefore, to compare their desorption energy obviating the size effect in equation (2), equilibrium interfacial coverage ratio $\Phi$ was calculated. This ratio $\Phi$ indicated the ratio of interfacial area covered by the two microgels when the exchanging particles established equilibrium with the energy input by the stirring. From the CLSM image in Figure 5, the areas occupied by each M30 particle and L30 particle on the interface were determined to be 0.72 and $1.65 \mu \mathrm{m}^{2}$, respectively. Therefore, $\Phi$ of M30 to L30 was determined to be 2.44, larger than 1. That meant for a given oil-water interface with a certain area, the adsorption of small microgel particles was more energetically favorable than the adsorption of the large microgel particles. Unfortunately, we could not quantify the difference of their desorption energy. It was because we could not quantify the energy which was given by the homogenizer. Nevertheless, this method provided an effective way to qualitatively compare the relative desorption energy of particles per unit area.

\section{Comparison of Interfacial Affinity Between Microgels With Different Cross-Linker Contents}

Similar to the previous comparison of surface affinity between microgels with different sizes, microgels with different crosslinker contents were mixed together and emulsions were prepared by the homogenizer. As we had five micron-sized microgel samples, the interfacial affinity of L10 was compared with L30, L50, L80, and L50A. Although the laser diffraction measurements of these samples were not exactly the same, their diameters in the CLSM images in Figure 6 were similar. Different types of the particles could mix with each other and achieve hexagonal packing on the interface. Table 4 summarizes the particle counting results. As each of these particles occupied almost the same area, we could compare their interfacial affinity simply having the number ratio equaled to the coverage ratio (i.e., $\varphi=\Phi)$.

According to the results in Table 4, when the cross-linker content of the microgel increased from $30 \mathrm{mg}$ (L30) to $80 \mathrm{mg}$

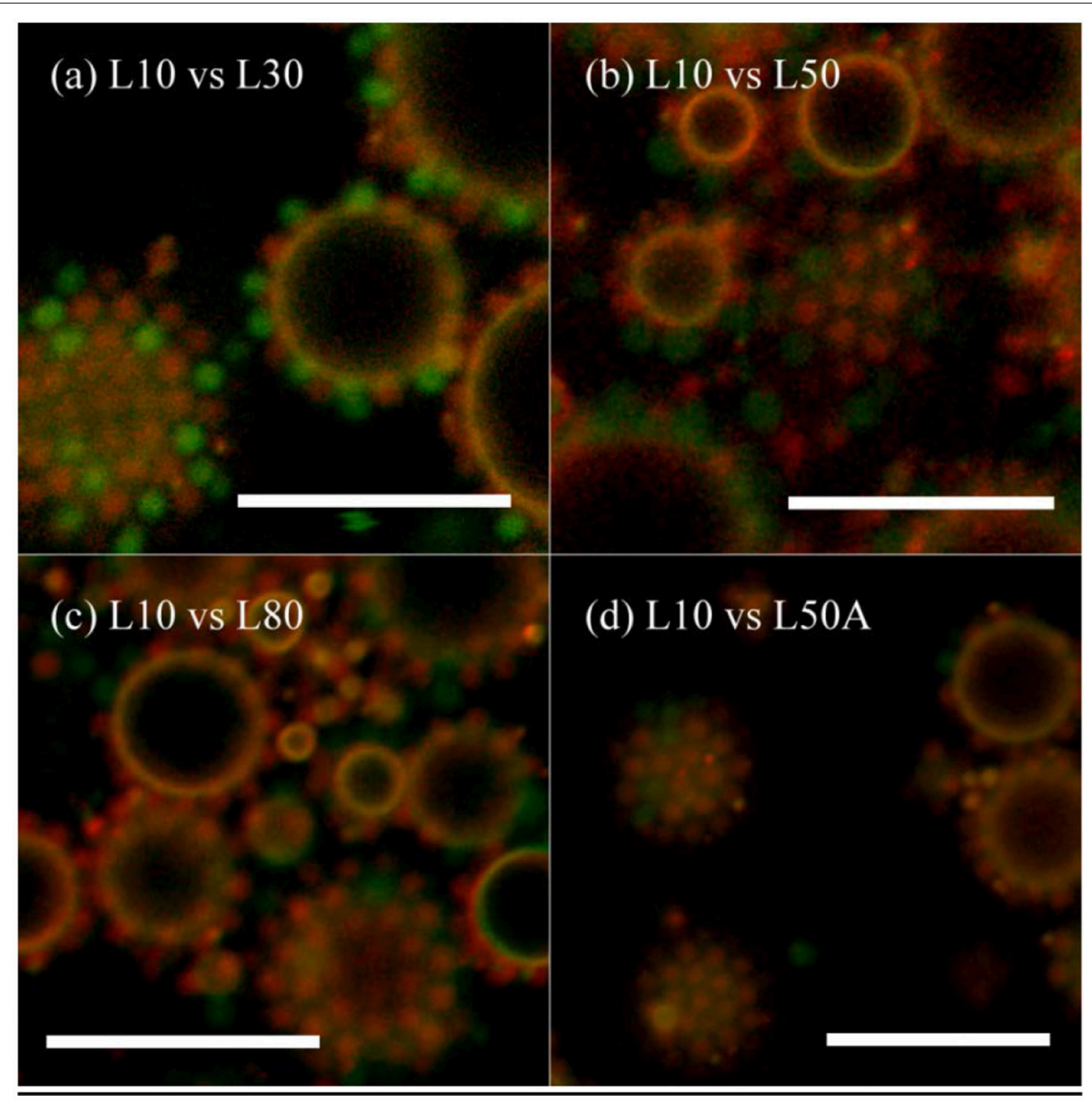

FIGURE 6 | CLSM image of emulsion prepared by L10 and other micron-sized microgels in 1:1 wt. conc. ratio. (a) L10 + L30, (b) L10 + L50, (c) L10 + L80, and (d) L10 + L50A. Red Rhodamine B fluorescence was only given by L10. Green fluorescein fluorescence was given both of the particles. The scale bars are all $10 \mu \mathrm{m}$. 
TABLE 4 | Particle counting results of large microgels at the interface.

\begin{tabular}{|c|c|c|c|c|c|c|c|c|c|}
\hline \multicolumn{10}{|c|}{ (A) L10 MIXED WITH L30 } \\
\hline Image no. & 1 & 2 & 3 & 4 & 5 & 6 & 7 & 8 & Total \\
\hline No. of L10 & 120 & 151 & 192 & 275 & 181 & 130 & 130 & 462 & 1,641 \\
\hline No. of L30 & 59 & 76 & 97 & 163 & 92 & 64 & 58 & 243 & 852 \\
\hline L10: L30 at interface & 2.03 & 1.99 & 1.98 & 1.69 & 1.97 & 2.03 & 2.24 & 1.90 & $1.93 \pm 0.15$ \\
\hline \multicolumn{10}{|c|}{ (B) L10 MIXED WITH L50 } \\
\hline Image no. & 1 & 2 & 3 & 4 & 5 & 6 & 7 & Total & \\
\hline No. of L10 & 318 & 345 & 434 & 243 & 245 & 342 & 295 & 2,222 & \\
\hline No. of L50 & 118 & 131 & 158 & 92 & 102 & 126 & 112 & 839 & \\
\hline L10: L50 at interface & 2.69 & 2.63 & 2.75 & 2.64 & 2.40 & 2.71 & 2.63 & $2.65 \pm 0.11$ & \\
\hline \multicolumn{10}{|c|}{ (C) L10 MIXED WITH L80 } \\
\hline Image no. & 1 & 2 & 3 & 4 & Total & & & & \\
\hline No. of L10 & 708 & 897 & 837 & 846 & 3,288 & & & & \\
\hline No. of L80 & 190 & 248 & 244 & 240 & 922 & & & & \\
\hline L10: L80 at interface & 3.73 & 3.62 & 3.43 & 3.53 & $3.57 \pm 0.12$ & & & & \\
\hline \multicolumn{10}{|c|}{ (D) L10 MIXED WITH L50A } \\
\hline Image no. & 1 & 2 & 3 & 4 & Total & & & & \\
\hline No. of L10 & 855 & 747 & 745 & 723 & 3,070 & & & & \\
\hline No. of L50A & 232 & 193 & 204 & 198 & 827 & & & & \\
\hline L10: L50A at interface & 3.69 & 3.87 & 3.65 & 3.65 & $3.71 \pm 0.10$ & & & & \\
\hline
\end{tabular}

The overall ratio is the weighted average of $\Phi$ and its weighted standard deviation.

(L80), their interfacial affinity relative to microgel with $10 \mathrm{mg}$ cross-linker (L10) decreased. More importantly, when we looked at the interfacial affinity of L50A relative to L10, it was smaller than that of L50 and L80. The calculated relative interfacial affinity is shown in Figure 7. The relative interfacial affinity is defined to be the reciprocal of the coverage ratio $\Phi$. Note that the relative interfacial affinity of L10 was by definition set to be 1 .

\section{Stability of Microgel Stabilized Pickering Emulsions: Effect of Cross-Linker Content}

Here, we focus the discussion on the effect of cross-linker content. The stabilities of the emulsions showed the same dependence on cross-linker content of microgel (L10, L30, L50 and L80). The stability of the resulting emulsion decreased as the cross-linking content of the stabilizing microgel increases. The main body of our micron-sized microgel particles (crosslinker content between 1.0 and $7.4 \% \mathrm{wt} / \mathrm{wt}$ ) were not significantly deforming at the interface. Therefore, we apply the conformation proposed by Geisel et al. in our discussion and focus at the periphery, the collapsed polymer layer at the interface (Geisel et al., 2012). Figure 8 shows a schematic illustration of the proposed conformation of microgel particle and the outermost collapsed polymer layer at the interface.

As mentioned above, in microgel batch synthesis, the crosslinker MBA was more reactive than the NIPAM monomer. As a result, the cross-linking density of the inner part of the microgel is higher. Also, the cross-linking density decreases gradually to the periphery of the particle. Here, we suggest that microgels with less cross-linker content also have a more deformable periphery. Therefore, they deformed more at the oil/water interface. Then, each of them was capable of covering and replacing more

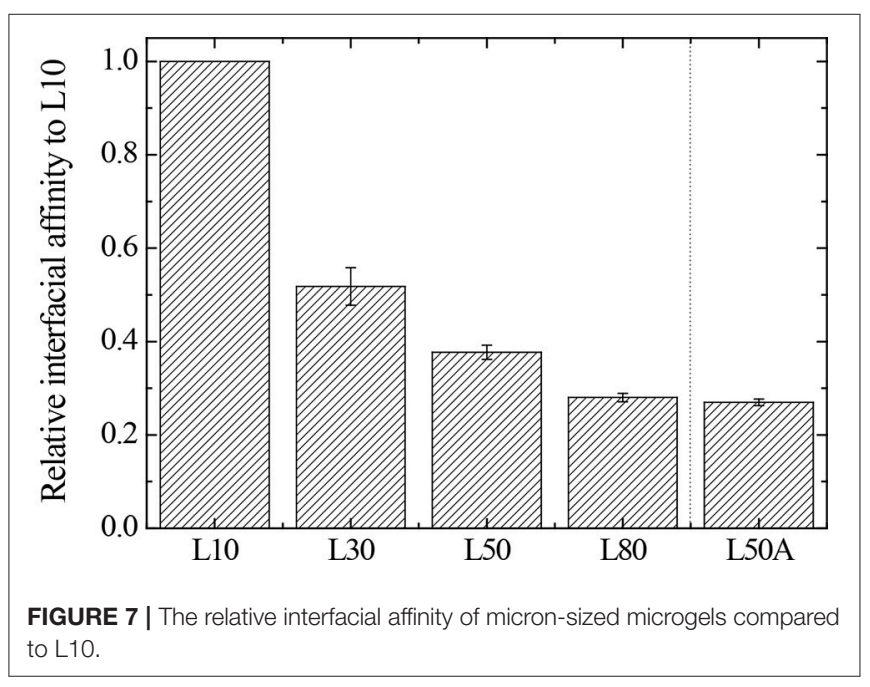

area at the interface. These outermost collapsed polymer chains on one hand act as anchors and help the particle attachment to the interface. On the other hand, because of low water content and the strong inter-and interapolymer interaction, the collapsed polymer layers at the interface also have a higher elastic modulus compared to the swollen microgels. As a resulting, the desorption energy of these particles, which has softer outer collapsed polymer layer, is higher.

In the previous section, the relative interfacial affinity was compared to the desorption energy of each of our microgel samples. Therefore, the desorption energy decreased from L10 to L80 gradually. It is consistent with our hypothesis and the stability measurement. 


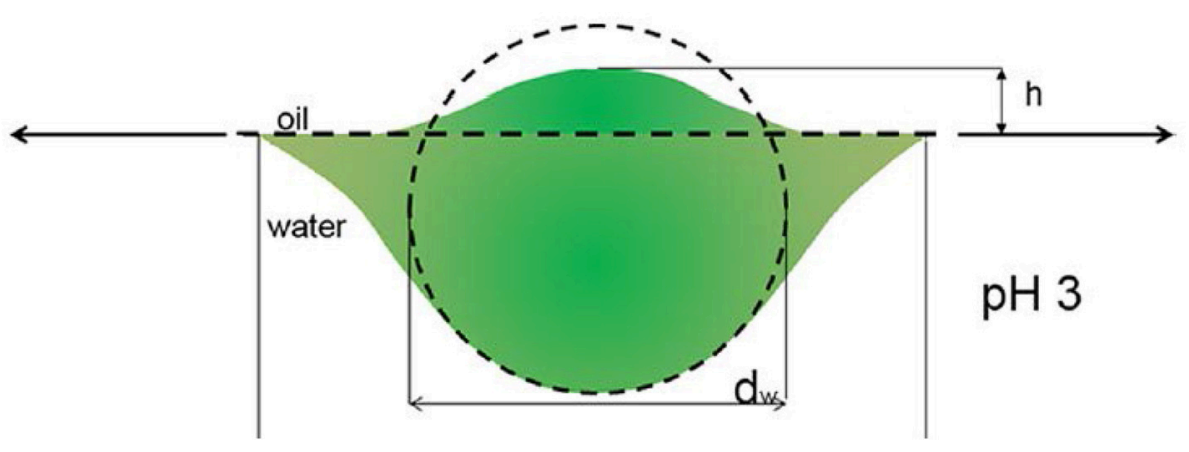

FIGURE 8 | The conformation of microgel particle at oil/water interface proposed by Geisel et al. (2012) Reprinted with permission from. Copyright (2012) American Chemical Society.

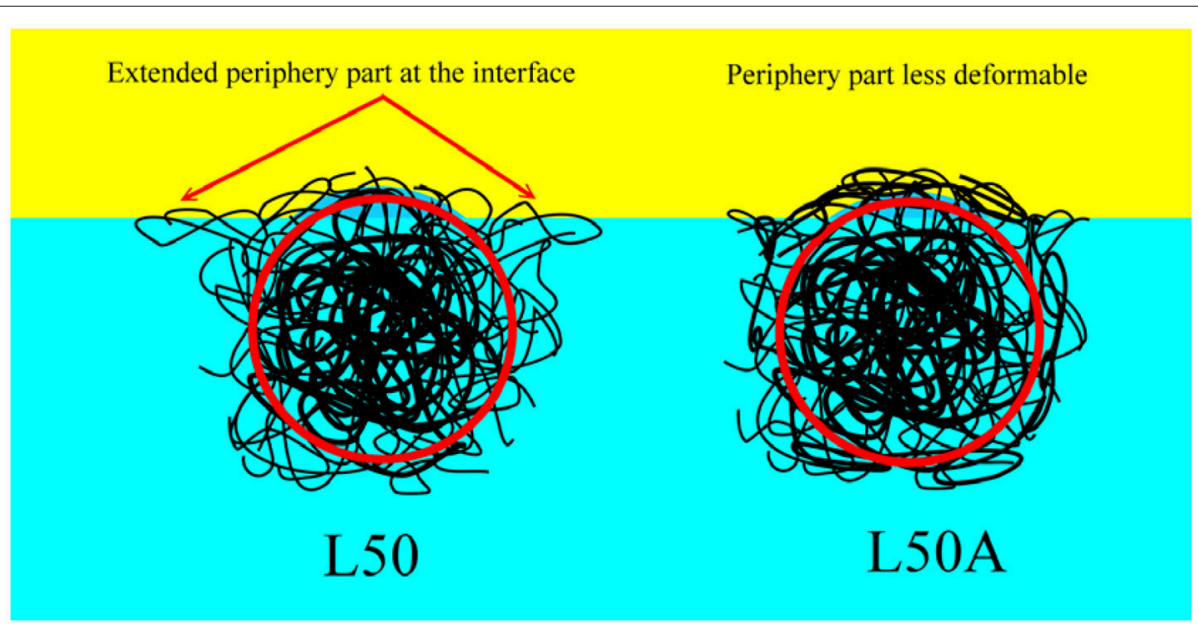

FIGURE 9 | The schematic illustrations of microgel L50 and L50A at oil/water interface. The red circles indicate the part which was labeled effectively by fluorescent dye. The soft periphery part and the main body of the microgel were not drawn in scale.

However, in order to verify this hypothesis, we prepared L50A. As mentioned above, the extra $10 \mathrm{mg}$ of cross-linker MBA was not involved in the particle growth at such low monomer concentrations. Therefore, it changed the microgel particle surface property by cross-linking some of the surface dangling chains. It decreased the deformability of the particle surface and outer portion collapsed polymer layer. It is very important to note that the overall deformability indicated by the thermo-responsive swelling ratios of L50 and L50A were very similar as they were synthesized by the same procedures. From the relative interfacial affinity results, the $10 \mathrm{mg}$ of extra crosslinker significantly reduced the interfacial affinity of the L50A microgel. It is important to point out that the overall crosslinker content of L80 was 33\% higher than that of L50A. These interfacial affinity results are also consistent with the stability measurements of the emulsions. In Figure 9, the schematic illustrations of microgel L50 and L50A at oil/water interface are shown. We show that if the outermost collapsed polymer layer portion of the particle was cross-linked, the desorption energy is lower compared to its counterpart, verifying our hypothesis.

\section{CONCLUSIONS}

We have demonstrated a new approach for comparing the relative interfacial affinity of soft colloids at the oil/water interface. Microgel samples demonstrated the good confocal image quality. By changing the preparation procedures and the amount of the microgels, it has been confirmed that the final ratio of interfacial particles is not kinetically controlled by the initial adsorption. Once the amount of oil is limited, the ratio is not affected by the amount of particles. Therefore, the interfacial coverage ratio of different microgels derived by this number ratio is capable of representing the relative affinity of the particles. The method was applied to study the effects of cross-linker content and surface deformability on the corresponding microgel-stabilized Pickering emulsions. It was found that microgels with less cross-linker content have higher interfacial affinity and better emulsion stability. Furthermore, the effect is more pronounced for the outermost collapsed polymer layer of the microgel. This result is consistent with the interfacial morphology proposed by other researches and 
provides direct connection between the deformability and the corresponding Pickering emulsion stability. The improvement in understanding the mechanism of soft colloids stabilized Pickering emulsions will be beneficial for further development of responsive Pickering emulsions with well-controlled stability and performance.

\section{AUTHOR CONTRIBUTIONS}

$\mathrm{TN}$ : conceived and managed the research. MK: performed the soft colloids synthesis, emulsion fabrication, and characterizations. TN and MK: reviewed the results and

\section{REFERENCES}

Aveyard, R., Binks, B., Fjetcher, P., and Rutherford, C. (1994). Contact angles in relation to the effects of solids on film and foam stability. J. Disp. Sci. Tech. 15, 251-271. doi: 10.1080/01932699408943557

Binks, B. P. (1998). "Emulsions-Recent advances in understanding," in Modern Aspects of Emulsion Science (Royal Society of Chemistry), 1-55.

Brugger, B., Rosen, B. A., and Richtering, W. (2008). Microgels as stimuliresponsive stabilizers for emulsions. Langmuir 24, 12202-12208. doi: $10.1021 / \mathrm{la} 8015854$

Brugger, B., Vermant, J., and Richtering, W. (2010). Interfacial layers of stimuliresponsive poly-(N-isopropylacrylamide-co-methacrylicacid)(PNIPAM-coMAA) microgels characterized by interfacial rheology and compression isotherms. Phys. Chem. Chem. Phys. 12, 14573-14578. doi: 10.1039/c0cp $01022 \mathrm{~g}$

Burmistrova, A., Richter, M., Uzum, C., and Klitzing, R. V. (2011). Effect of cross-linker density of P (NIPAM-co-AAc) microgels at solid surfaces on the swelling/shrinking behaviour and the Young's modulus. Colloid Polym. Sci. 289, 613-624. doi: 10.1007/s00396-011-2383-2

Chen, T., Colver, P. J., and Bon, S. A. (2007). Organic-inorganic hybrid hollow spheres prepared from $\mathrm{TiO}_{2}$-stabilized pickering emulsion polymerization. Adv. Mater. 19, 2286-2289. doi: 10.1002/adma.200602447

Chevalier, Y., and Bolzinger, M.-A. (2013). Emulsions stabilized with solid nanoparticles: Pickering emulsions. Colloids Surf. A 439, 23-34. doi: 10.1016/j.colsurfa.2013.02.054

Destribats, M., Eyharts, M., Lapeyre, V., Sellier, E., Varga, I., Ravaine, V., et al. (2014). Impact of pNIPAM microgel size on its ability to stabilize Pickering emulsions. Langmuir 30, 1768-1777. doi: 10.1021/la4044396

Destribats, M., Lapeyre, V., Wolfs, M., Sellier, E., Leal-Calderon, F., Ravaine, V., et al. (2011). Soft microgels as Pickering emulsion stabilisers: role of particle deformability. Soft Matter 7, 7689-7698. doi: 10.1039/c1sm05240c

Destribats, M., Wolfs, M., Pinaud, F., Lapeyre, V., Sellier, E., Schmitt, V., et al. (2013). Pickering emulsions stabilized by soft microgels: influence of the emulsification process on particle interfacial organization and emulsion properties. Langmuir 29, 12367-12374. doi: 10.1021/la402921b

Frelichowska, J., Bolzinger, M. A., Pelletier, J., Valour, J. P., and Chevalier, Y. (2009). Topical delivery of lipophilic drugs from o/w Pickering emulsions. Int. J. Pharm. 371, 56-63. doi: 10.1016/j.ijpharm.2008.12.017

Geisel, K., Henzler, K., Guttmann, P., and Richtering, W. (2014a). New insight into microgel-stabilized emulsions using transmission X-ray microscopy: nonuniform deformation and arrangement of microgels at liquid interfaces. Langmuir 31, 83-89. doi: 10.1021/la503959n

Geisel, K., Isa, L., and Richtering, W. (2012). Unraveling the 3D localization and deformation of responsive microgels at oil/water interfaces: a step forward in understanding soft emulsion stabilizers. Langmuir 28, 15770-15776. doi: $10.1021 /$ la 302974 j

Geisel, K., Richtering, W., and Isa, L. (2014b). Highly ordered 2D microgel arrays: compression versus self-assembly. Soft Matter 10, 7968-7976. doi: 10.1039/C4SM01166J provided the technical guidelines. $\mathrm{TN}$ and $\mathrm{MK}$ : wrote and drafted the article. TN and MK: reviewed and approved the article.

\section{ACKNOWLEDGMENTS}

The financial support of this work by the Hong Kong Special Administration Region (HKSAR) General Research Fund (CUHK14306617, 2130535), the National Natural Science Foundation of China (21574110), and the Direct Grant for Research (3132681, 3132682, and 4053109) of the Chinese University of Hong Kong is gratefully acknowledged.

Hashmi, S. M., and Dufresne, E. R. (2009). Mechanical properties of individual microgel particles through the deswelling transition. Soft Matter 5, 3682-3688. doi: 10.1039/b906051k

Hoare, T., and Pelton, R. (2004). Highly $\mathrm{pH}$ and temperature responsive microgels functionalized with vinylacetic acid. Macromolecules 37, 2544-2550. doi: $10.1021 / \mathrm{ma} 035658 \mathrm{~m}$

Hoare, T., and Pelton, R. (2008). Characterizing charge and crosslinker distributions in polyelectrolyte microgels. Curr. Opin. Colloid Interface Sci. 13, 413-428. doi: 10.1016/j.cocis.2008.03.004

Khan, A. (2007). Preparation and characterization of Nisopropylacrylamide/acrylic acid copolymer core-shell microgel particles. J. Colloid Interface Sci. 313, 697-704. doi: 10.1016/j.jcis.2007.05.027

Khan, A. (2008). Preparation and characterization of magnetic nanoparticles embedded in microgels. Mater. Lett. 62, 898-902. doi: 10.1016/j.matlet.2007.07.011

Kwok, M. H., Li, Z., and Ngai, T. (2013). Controlling the synthesis and characterization of micrometer-sized PNIPAM microgels with tailored morphologies. Langmuir 29, 9581-9591. doi: 10.1021/la402062t

Kwok, M. H., and Ngai, T. (2016). A confocal microscopy study of micron-sized poly (N-isopropylacrylamide) microgel particles at the oil-water interface and anisotopic flattening of highly swollen microgel. J. Colloid Interface Sci. 461, 409-418. doi: 10.1016/j.jcis.2015.09.049

Li, J., and Stöver, H. D. (2008). Doubly pH-responsive pickering emulsion. Langmuir 24, 13237-13240. doi: 10.1021/la802619m

Liu, B., Wei, W., Qu, X., and Yang, Z. (2008). Janus colloids formed by biphasic grafting at a Pickering emulsion interface. Angew. Chem. 120, 4037-4039. doi: 10.1002/ange.200705103

Monteillet, H., Workamp, M., Appel, J., Kleijn, J. M., Leermakers, F. A., and Sprakel, J. (2014). Ultrastrong anchoring yet barrier-free adsorption of composite microgels at liquid interfaces. Adv. Mater. Interfaces 1:1300121. doi: 10.1002/admi.201300121

Ngai, T., Auweter, H., and Behrens, S. H. (2006). Environmental responsiveness of microgel particles and particle-stabilized emulsions. Macromolecules 39, 8171-8177. doi: 10.1021/ma061366k

Ngai, T., Behrens, S. H., and Auweter, H. (2005). Novel emulsions stabilized by $\mathrm{pH}$ and temperature sensitive microgels. Chem. Commun. 331-333. doi: $10.1039 / \mathrm{b} 412330 \mathrm{a}$

Pelton, R., and Chibante, P. (1986). Preparation of aqueous latices with N-isopropylacrylamide. Colloids Surf. 20, 247-256. doi: 10.1016/0166-6622(86)80274-8

Pickering, S. U. (1907). Cxcvi.-emulsions. J. Chem. Soc. Trans. 91, 2001-2021. doi: 10.1039/CT9079102001

Pinaud, F., Geisel, K., Massé, P., Catargi, B., Isa, L., Richtering, W., et al. (2014). Adsorption of microgels at an oil-water interface: correlation between packing and 2D elasticity. Soft Matter 10, 6963-6974. doi: 10.1039/C4SM00562G

Ramsden, W. (1904). Separation of solids in the surface-layers of solutions and 'suspensions'(observations on surface-membranes, bubbles, emulsions, and mechanical coagulation).-Preliminary account. Proc. R. Soc. Lond. 72, 156-164. doi: 10.1098/rspl.1903.0034 
Richtering, W. (2012). Responsive emulsions stabilized by stimuli-sensitive microgels: emulsions with special non-Pickering properties. Langmuir 28, 17218-17229. doi: 10.1021/la302331s

Saunders, B. R., and Vincent, B. (1999). Microgel particles as model colloids: theory, properties and applications. Adv. Colloid Int. Sci. 80, 1-25. doi: 10.1016/S0001-8686(98)00071-2

Schmitt, V., and Ravaine, V. (2013). Surface compaction versus stretching in Pickering emulsions stabilised by microgels. Curr. Opin. Colloid Int. Sci. 18, 532-541. doi: 10.1016/j.cocis.2013.11.004

Style, R. W., Isa, L., and Dufresne, E. R. (2015). Adsorption of soft particles at fluid interfaces. Soft Matter 11, 7412-7419. doi: 10.1039/C5SM $01743 \mathrm{~B}$

Tang, J., Quinlan, P. J., and Tam, K. C. (2015). Stimuli-responsive Pickering emulsions: recent advances and potential applications. Soft Matter 11, 3512-3529. doi: 10.1039/C5SM00247H

Tcholakova, S., Denkov, N. D., Ivanov, I. B., and Campbell, B. (2002). Coalescence in $\beta$-lactoglobulin-stabilized emulsions: effects of protein adsorption and drop size. Langmuir 18, 8960-8971. doi: 10.1021/la02 58188

Tsuji, S., and Kawaguchi, H. (2008). Thermosensitive Pickering emulsion stabilized by poly (N-isopropylacrylamide)-carrying particles. Langmuir 24, 3300-3305. doi: $10.1021 / \operatorname{la} 701780 \mathrm{~g}$
Wiese, S., Spiess, A. C., and Richtering, W. (2013). Microgel-Stabilized Smart Emulsions for Biocatalysis. Angew. Chem. 125, 604-607. doi: 10.1002/ange.201206931

Zhang, K., Wu, W., Guo, K., Chen, J., and Zhang, P. (2010). Synthesis of temperature-responsive poly (N-isopropyl acrylamide)/poly (methyl methacrylate)/silica hybrid capsules from inverse pickering emulsion polymerization and their application in controlled drug release. Langmuir 26, 7971-7980. doi: 10.1021/la904841m

Zielinska, K., Sun, H., Campbell, R. A., Zarbakhsh, A., and Resmini, M. (2016). Smart nanogels at the air/water interface: structural studies by neutron reflectivity. Nanoscale 8, 4951-4960. doi: 10.1039/C5NR07538F

Conflict of Interest Statement: The authors declare that the research was conducted in the absence of any commercial or financial relationships that could be construed as a potential conflict of interest.

Copyright (C) 2018 Kwok and Ngai. This is an open-access article distributed under the terms of the Creative Commons Attribution License (CC BY). The use, distribution or reproduction in other forums is permitted, provided the original author(s) and the copyright owner are credited and that the original publication in this journal is cited, in accordance with accepted academic practice. No use, distribution or reproduction is permitted which does not comply with these terms. 